\title{
Late regression of left internal thoracic artery graft stenosis at the anastomotic site without intervention therapy
}

Chisato Izumi, MD, ${ }^{a}$ Hidetaka Hayashi, ${ }^{\mathrm{b}}$ Yuichi Ueda, MD, ${ }^{\mathrm{c}}$ Masahiko Matsumoto, MD, ${ }^{\mathrm{d}}$ Yoshihiro Himura, MD, ${ }^{\text {a }}$ Hiromitsu Gen, MD, and Takashi Konishi, MD ${ }^{\mathrm{a}}$

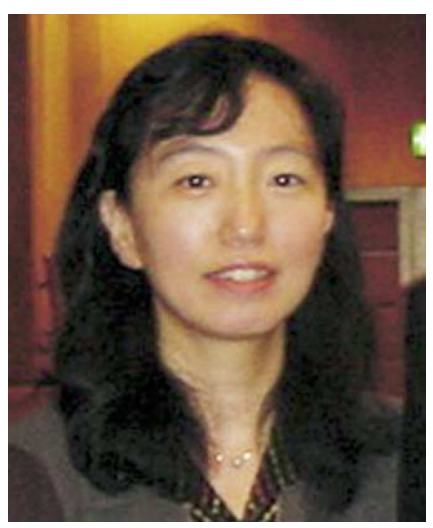

Dr Izumi

See related editorial on page 1494.
From the Departments of Cardiology, ${ }^{\text {a }}$ Radiology, ${ }^{\mathrm{b}}$ and Cardiovascular Surgery, ${ }^{\mathrm{d}}$ Tenri Hospital, Nara, Japan, and the Department of Thoracic Surgery, ${ }^{c}$ Nagoya University, Nagoya, Japan.

Received for publication Feb 21, 2005; revisions recived July 7, 2005; accepted for publication July 19, 2005.

Address for reprints: Chisato Izumi, MD, Department of Cardiology, Tenri Hospital, 200 Mishima-cho, Tenri-city, Nara, 632, Japan (E-mail: izumi-ch@tenriyorozu-hp.or.jp).

J Thorac Cardiovasc Surg 2005;130:1661-7

0022-5223/\$30.00

Copyright (C) 2005 by The American Association for Thoracic Surgery

doi:10.1016/j.jtcvs.2005.07.019
Objective: Intervention therapy has been recently performed on the left internal thoracic artery graft stenosis. The purpose of this study was to evaluate the natural course of the left internal thoracic artery graft stenosis at the anastomotic site and clarify whether intervention therapy should be performed early after surgery.

Methods: We investigated early angiographic results of the left internal thoracic artery graft in 343 consecutive patients who underwent coronary bypass surgery. In 100 of 343 patients who underwent follow-up angiography, the graft diameter and percentage diameter stenosis at the anastomotic site were compared between early postoperative and follow-up angiography. None of these patients underwent intervention therapy on the left internal thoracic artery graft.

Results: Of 343 patients, 46 showed $50 \%$ or greater diameter stenosis, and 20 showed $70 \%$ or greater diameter stenosis at the anastomotic site. In the 100 patients with follow-up angiography, the graft diameter significantly increased (1.8 $\pm 0.4 \mathrm{vs}$ $2.1 \pm 0.5 \mathrm{~mm}, P<.0001)$ at follow-up angiography. The percentage diameter stenosis significantly decreased $(69 \% \pm 13 \%$ vs $35 \% \pm 20 \%, P<.0001)$ at follow-up angiography in the patients with $50 \%$ or greater diameter stenosis at early postoperative angiography. Regression of left internal thoracic artery graft stenosis was detected in most patients with $70 \%$ or greater diameter stenosis.

Conclusions: Our study demonstrated that left internal thoracic artery graft stenosis at the anastomotic site at early postoperative angiography might improve without intervention therapy. We should consider the natural course of the left internal thoracic artery graft stenosis in determining the indication of intervention therapy early after surgery.

$\mathrm{T}$ The left internal thoracic artery (LITA) has been widely used as a graft for coronary bypass surgery because of its superior long-term patency, ${ }^{1,2}$ but graft stenosis is sometimes detected at the anastomotic site at early postoperative angiography. Intervention therapy has been recently performed on the LITA graft stenosis at the anastomotic site, and results of the intervention therapy have been reported to be good. ${ }^{3,4}$ However, there are few reports about the natural course of the focal stenosis of the LITA graft at the anastomotic site. Previous studies have demonstrated that edema or hematoma at the anastomotic site might be induced by operative procedures or that the LITA graft might easily become spastic early after the operation. ${ }^{5-10}$ The purpose of this study is to evaluate the natural course of the LITA graft stenosis at the anastomotic site and to clarify whether intervention therapy should be performed on the LITA graft stenosis early after surgery. 


\section{Abbreviations and Acronyms}

LITA $=$ left internal thoracic artery

\section{Methods \\ Study Patients}

Among 430 patients who underwent coronary bypass surgery with the LITA graft between May 1988 and February 2000 in Tenri Hospital, we retrospectively investigated early results of the LITA graft in 343 consecutive patients ( 257 male and 86 female patients; age, $64 \pm 9$ years; age range, 24-82 years) who underwent postoperative angiography early after surgery. We referred to clinical and angiographic examination records of these patients, and 100 of 343 patients turned out to have undergone follow-up angiography of the LITA graft. We retrospectively investigated changes in the diameter of the LITA graft and the degree of the LITA graft stenosis in these 100 patients (79 male and 21 female patients; age, $60 \pm 8$ years; age range, 40-76 years). None of them underwent intervention therapy on the LITA graft. Early postoperative angiography was performed about 3 weeks after surgery, and follow-up angiography was performed at an average of 5 years after surgery (range, 5 months to 15 years). Follow-up angiography was performed for evaluating new-onset angina pectoris in 70 patients, congestive heart failure in 12 patients, acute myocardial infarction in 6 patients, and aortic valve stenosis in 3 patients and for a follow-up study without symptoms in 9 patients.

During this study, 3 staff surgeons performed coronary bypass surgery. The surgical technique was basically the same among the surgeons. Technical details were as follows. The LITA was harvested along the full length from the sixth intercostal space to the first rib. The pedicle was wrapped in a papaverine-soaked gauze. When the end of the LITA was being prepared, it was cut in a beveled fashion so that the circumference of the LITA opening was $10 \%$ to $20 \%$ larger than that of the opening in the coronary artery, providing a large hood of the LITA over the distal anastomosis. The anterior wall of the coronary artery was opened longitudinally. The incision was enlarged to a length of about $5 \mathrm{~mm}$, approximately two and a half times longer than the diameter of the coronary artery. The technique of anastomosis used one doublearmed 7-0 polypropylene suture placed as a continuous stitch. Stitches in the LITA went from outside to inside, and those in the coronary artery went from inside to outside. Suture bites in the heel of the LITA were about $0.5 \mathrm{~mm}$ in depth.

The site of anastomosis was the middle portion or distal portion of the left anterior descending artery in 319 patients, the diagonal branch in 10 patients, and the obtuse marginal branch or posterolateral branch of the left circumflex artery in 14 patients. One hundred thirty-five of 343 patients had a previous myocardial infarction in the area of the coronary artery to which the LITA graft was anastomosed.

The degree of native coronary artery flow at early postoperative and follow-up angiography was classified into 3 categories: wellpreserved flow, poorly preserved flow, and no flow. Native coronary angiography was not performed in 3 patients at early postoperative angiography and 4 patients at follow-up angiography. At early postoperative angiography, 141 patients showed well-

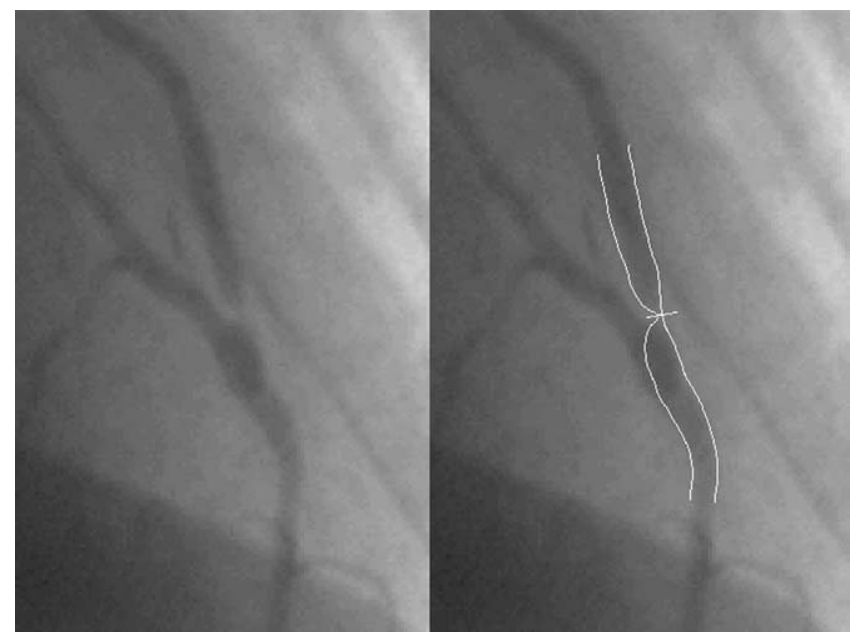

Figure 1. Measurement of percentage diameter stenosis at the anastomotic site by means of quantitative angiographic analysis.

preserved, 69 patients showed poorly preserved, and 130 patients showed no native coronary artery flow. At follow-up angiography in 100 patients, 26 patients showed well-preserved, 14 patients showed poorly preserved, and 56 patients showed no native coronary artery flow. Native coronary artery flow decreased at follow-up angiography in a total of 18 of the 100 patients. Informed consent was obtained from all patients. The study protocol was approved by the ethics committee at Tenri Hospital.

\section{Angiographic Analysis of the LITA Graft}

The LITA graft was examined by multiple projections after intravenous administration of $1 \mathrm{mg}$ of isosorbide dinitrate. Quantitative angiographic analysis was performed with an automated edgedetection algorithm (CASS II, Pie Medical Imaging). The diameter of a $5 \mathrm{~F}$ catheter at the LITA ostium was used as the calibration standard. The diameter of the LITA graft and percentage diameter stenosis at the anastomotic site were measured by means of quantitative angiography (Figure 1). In 100 patients who underwent follow-up angiography, the diameter of the LITA graft and percentage diameter stenosis at the anastomotic site were compared between early postoperative and follow-up angiography. A normal-appearing segment of 1.5 to $2 \mathrm{~cm}$ in length near the anastomotic site was chosen for measurement of the diameter of the LITA graft. Native coronary artery diameter near the anastomotic site was also measured. The percentage diameter stenosis was calculated from a magnified image obtained in the most stenotic view at early postoperative angiography and in the same view at follow-up angiography.

String sign was defined as a patent LITA graft with less than 1 $\mathrm{mm}$ diameter without focal stenosis.

\section{Statistical Analysis}

Values were expressed as means \pm standard deviation. Differences in the parameters between early postoperative and follow-up angiography were determined by using paired $t$ test analysis. Differences in the parameters between 2 groups were determined by 
TABLE 1. Early results of the LITA graft and patient numbers with follow-up angiography

\begin{tabular}{lrcr}
\hline & \multicolumn{2}{c}{$\begin{array}{c}\text { Early postoperative } \\
\text { angiography }\end{array}$} & \\
\cline { 2 - 3 } & $\mathbf{n}$ & $\begin{array}{c}\text { Diameter of the } \\
\text { LITA graft (mm) }\end{array}$ & \multicolumn{1}{c}{ Follow-up } \\
\hline$<50 \%$ stenosis & 264 & $1.8 \pm 0.3$ & 64 \\
$50 \%-69 \%$ stenosis & 26 & $1.7 \pm 0.3$ & 12 \\
$\geq 70 \%$ stenosis & 20 & $1.7 \pm 0.3$ & 10 \\
String sign & 18 & $0.8 \pm 0.1$ & 6 \\
Occlusion & 15 & & 8
\end{tabular}

LITA, Left internal thoracic artery.

using unpaired $t$ test analysis. The differences in a parameter between 2 groups were determined by using the Fisher exact test for discrete variables. Regression analysis was used to assess the correlation between 2 parameters.

\section{Results}

\section{Early Results of the LITA Graft}

Among 343 patients, 264 showed less than 50\% diameter stenosis $(15 \% \pm 14 \%$ diameter stenosis, with no stenosis in 171 patients), 26 patients showed $50 \%$ to $69 \%$ diameter stenosis, and 20 patients showed $70 \%$ or greater diameter stenosis $(70 \%-79 \%$ diameter stenosis in 11 patients, $80 \%$ $89 \%$ diameter stenosis in 7 patients, and $\geq 90 \%$ diameter stenosis in 2 patients) at the anastomotic site (Table 1). Eighteen patients showed string sign, and 15 patients showed occlusion of the LITA graft. The diameter of the LITA graft was $1.8 \pm 0.3 \mathrm{~mm}$ in 264 patients with less than $50 \%$ diameter stenosis, $1.7 \pm 0.3 \mathrm{~mm}$ in 26 patients with $50 \%$ to $69 \%$ diameter stenosis, $1.7 \pm 0.3 \mathrm{~mm}$ in 20 patients with $70 \%$ or greater diameter stenosis, and $0.8 \pm 0.1 \mathrm{~mm}$ in 18 patients with the string sign (Table 1).
The diameter of the LITA graft revealed a weak correlation with the diameter of the native coronary artery at early postoperative angiography $(r=0.37, P<.0001)$. The diameter of the LITA graft in the patients with wellpreserved native coronary artery flow was significantly smaller than that in the patients with poorly preserved $(1.6 \pm 0.4 \mathrm{~mm}$ [well preserved] vs $1.8 \pm 0.3 \mathrm{~mm}$ [poorly preserved]; $P=$ $.0012)$ and no $(1.6 \pm 0.4 \mathrm{~mm}$ [well preserved] vs $1.9 \pm 0.3$ $\mathrm{mm}$ [no flow]; $P<.0001)$ native coronary artery flow. The percentage diameter stenosis at the anastomotic site had a very weak negative correlation with the diameter of the LITA graft $(r=-0.17, P=.0028)$. The degree of native coronary artery flow showed no relationship with the presence of $50 \%$ or greater diameter stenosis at the anastomotic site.

\section{Comparison Between Early Postoperative and Follow-up Angiography}

Follow-up angiography was performed in 100 patients in total: 64 of 264 patients with less than $50 \%$ diameter stenosis, 12 of 26 patients with $50 \%$ to $69 \%$ diameter stenosis, 10 of 20 patients with $70 \%$ or greater diameter stenosis, 6 of 18 patients with the string sign, and 8 of 15 patients with an occluded LITA graft at early postoperative angiography (Table 1).

In the 6 patients with string sign at early postoperative angiography, the LITA graft was occluded in 4 patients, the string sign was unchanged in 1 patient, and the string sign disappeared in 1 patient at follow-up angiography. The LITA graft remained occluded at follow-up angiography in the 8 patients who showed occlusion of the LITA graft at early postoperative angiography.

In the remaining 86 patients with follow-up angiography, the diameter of the LITA graft was significantly larger (1.8 \pm 0.4 vs $2.1 \pm 0.5 \mathrm{~mm}, P<.0001)$, and percentage diameter stenosis was significantly decreased $(32 \% \pm 27 \%$ vs $21 \% \pm$

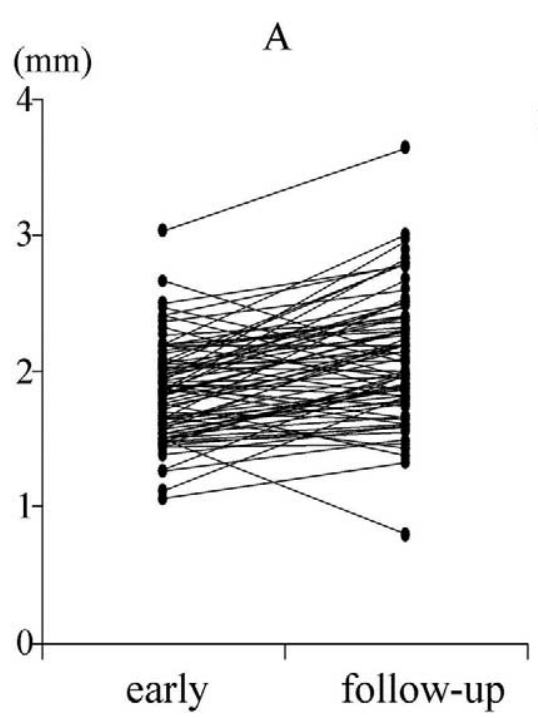

\section{(\%)}

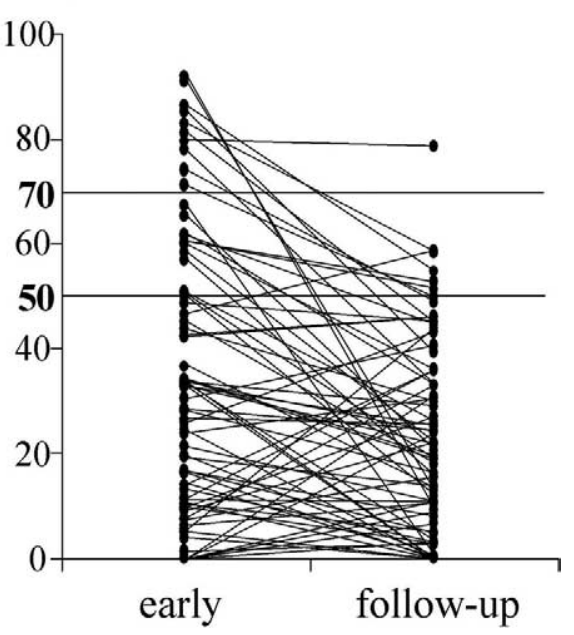

Figure 2. The diameter of the left internal thoracic artery graft $(A)$ and percentage diameter stenosis at the anastomotic site (B) at early postoperative and follow-up angiography. 


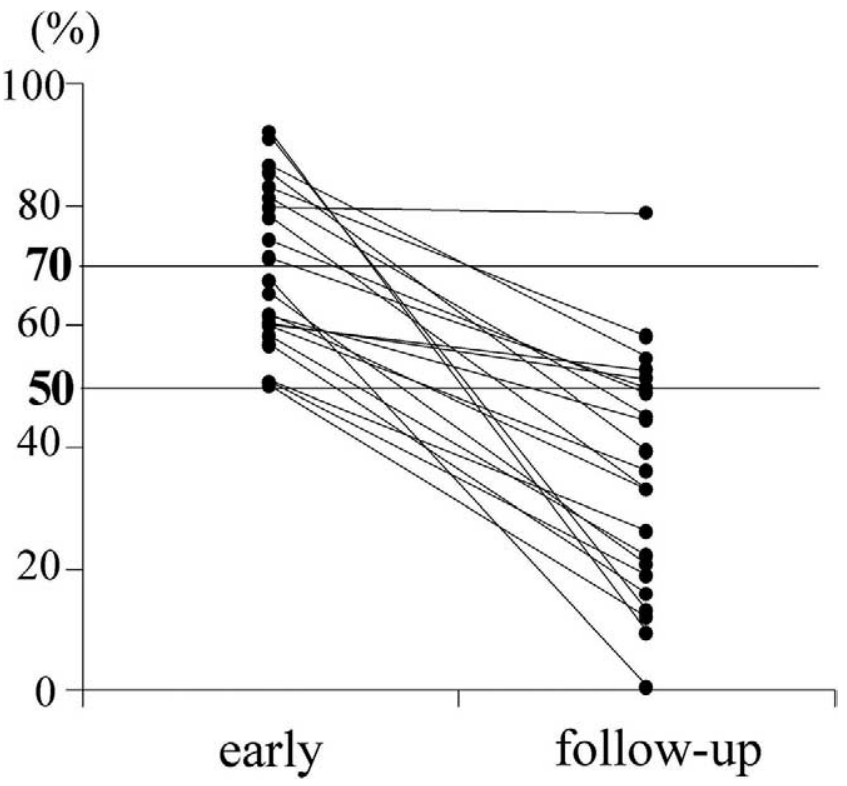

Figure 3. The change in percentage diameter stenosis of the left internal thoracic artery graft in the patients with $\mathbf{5 0} \%$ or greater diameter stenosis at early postoperative angiography.

$19 \%, P<.0001)$ at follow-up angiography compared with that at early postoperative angiography (Figure 2). String sign developed at follow-up angiography in 1 patient. Only 1 of 64 patients with less than $50 \%$ diameter stenosis at early postoperative angiography showed $50 \%$ or greater diameter stenosis at follow-up angiography.

In 22 patients with $50 \%$ or greater diameter stenosis at early postoperative angiography, the percentage diameter stenosis significantly decreased at follow-up angiography $(69 \% \pm 13$ vs $35 \% \pm 20 \%, P<.0001$; Figure 3$)$. Among 10 patients with $70 \%$ or greater diameter stenosis at early postoperative angiography, regression of the LITA graft stenosis was detected in 9 patients without intervention therapy (Figure 3).

Follow-up angiography in 10 patients who showed $70 \%$ or greater diameter stenosis at early postoperative angiography was performed for evaluating new-onset angina pectoris in 7 patients, acute myocardial infarction in 2 patients, and a follow-up study without symptoms in 1 patient. Four of 10 patients underwent intervention therapy on vessels other than the LITA graft, but they did not show significant stenosis of the LITA graft at that time.

A representative case is shown in Figure 4.

\section{Detection of Ischemia in Patients With LITA Graft Stenosis}

All patients with $50 \%$ or greater diameter stenosis at the anastomotic site were asymptomatic early after surgery.
Thallium-201 stress scintigraphy was performed in 15 of 20 patients with $70 \%$ or greater diameter stenosis early after surgery. Heart rate increased to $117 \pm 22$ beats $/ \min (76 \% \pm$ $12 \%$ of the age-predicted maximal heart rate) at peak stress. Seven patients showed normal perfusion image, 6 patients showed fixed defect, and 2 patients showed partial redistribution in the area of the coronary artery to which the LITA graft was anastomosed. The 2 patients who showed redistribution did not need intervention therapy because redistribution was detected in the small area of the diagonal branch and the first septal branch, respectively.

\section{Long-Term Prognosis of the Patients With 70\% or Greater Diameter Stenosis at Early Postoperative Angiography}

The mean follow-up period was $124 \pm 42$ months after coronary bypass surgery. Among 20 patients with $70 \%$ or greater diameter stenosis early after surgery, 5 patients died during follow-up, and death occurred at a mean of 101 months after surgery. Among the 5 patients, 3 patients showed $70 \%$ to $79 \%$ diameter stenosis and 2 patients showed $80 \%$ to $89 \%$ diameter stenosis at early postoperative angiography. Cause of death was sudden death in 3 patients, congestive heart failure in 1 patient, and sepsis in 1 patient. All 5 patients underwent follow-up angiography, and all but 1 patient showed regression of the LITA graft stenosis. One patient who did not show regression of the LITA graft stenosis at follow-up angiography had sudden death 134 months after surgery. Two patients with $90 \%$ or greater diameter stenosis early after surgery have been doing well without any cardiac events. No patients required intervention therapy on the LITA graft late after surgery.

\section{Discussion}

\section{Previous Studies}

The LITA has been widely used as a graft for coronary bypass surgery because of its long-term patency. ${ }^{1,2}$ However, there are some issues, including inadequate perfusion during and early after surgery ${ }^{9,10}$ and string sign, which is diffuse narrowing or uniform distal narrowing of the LITA graft. ${ }^{11-13}$ The causes of string sign have not been determined, but some possible mechanisms were mentioned in previous studies. ${ }^{8,11,13-16}$ Graft injury during the operation, intimal proliferation, blood flow competition between the LITA graft and recipient coronary artery, blood flow steal phenomenon caused by side branches of the LITA, spasm, and edema caused by surrounding inflammation are possible mechanisms of the string sign. The LITA graft has been reported to be a physiologically active conduit, and the diameter of the LITA graft might change, depending on the degree of native coronary artery flow. ${ }^{11,17}$ Spontaneous recanalization of the occluded LITA graft was also reported, which was associated with progression of the native coronary artery stenosis. ${ }^{14-16}$ 


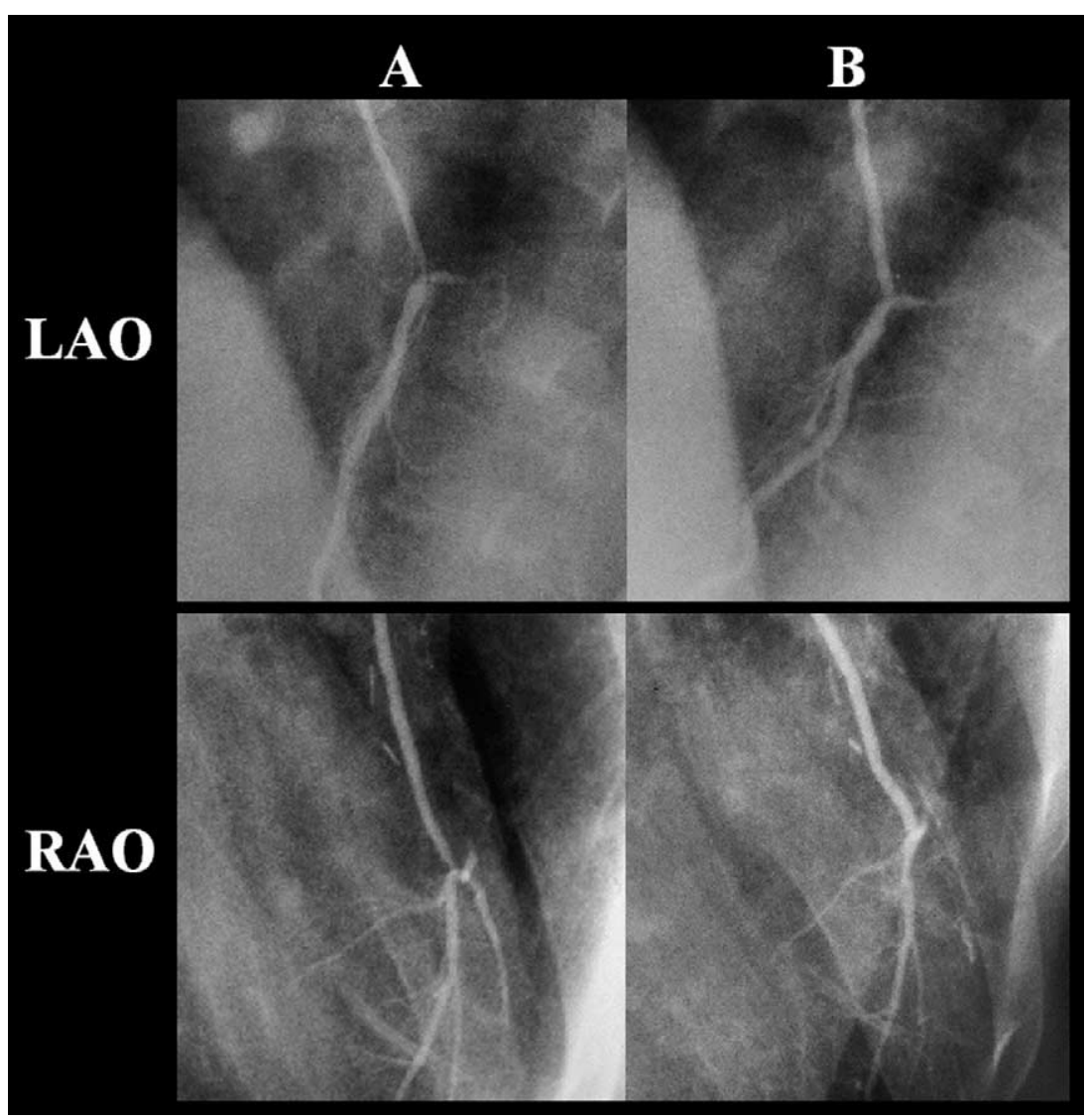

Figure 4. A representative case. This case showed $85 \%$ diameter stenosis at early postoperative angiography (A). Stenosis at the anastomotic site disappeared at follow-up angiography $(B)$ without intervention therapy. LAO, Left anterior oblique view; RAO, right anterior oblique view.

Berger and colleagues ${ }^{18}$ demonstrated that early patency of the LITA graft was more than $98 \%$, but $7.8 \%$ of the patients had $50 \%$ or greater stenosis within the LITA graft, and $1.3 \%$ of the patients showed occlusion of the LITA graft. The LITA graft stenosis is sometimes detected at the anastomotic site at early postoperative angiography, and intervention therapy has been recently performed on the LITA graft stenosis at the anastomotic site. ${ }^{3,4}$ However, there are few reports about the natural course of the focal stenosis of the LITA graft at the anastomotic site.

\section{Mechanisms of LITA Graft Stenosis at the Anastomotic Site}

In this study we focused on the focal stenosis at the anastomotic site, and regression of the LITA graft stenosis was detected in most patients without intervention therapy. This finding suggested that the LITA graft stenosis detected at early postoperative angiography was transient stenosis in most patients. Therefore we believe that surgical technical failure was not a main cause of the LITA graft stenosis at the anastomotic site in our study. We speculate that edema caused by operative procedures, hematoma, or spasm around the anastomotic site might be one of the causes of the transient stenosis at the anastomotic site. A thrombus at the anastomotic site might be a possible mechanism of the transient stenosis, but an intraluminal filling defect that was suggestive of a thrombus was not observed at early postoperative angiography. Natural healing of local dissection of the LITA graft or remodeling might be a possible mechanism of regression of the LITA graft stenosis, but we have no data about it because an intravascular ultrasound study was not performed.

There is a possibility that the LITA graft stenosis at early postoperative angiography is not rigid and is expandable, and the degree of the stenosis might change depending on the LITA graft flow. Although we could not evaluate the LITA graft flow itself by using a Doppler flow wire in the present study, the diameter of the LITA graft and the degree of stenosis at the anastomotic site showed a weak negative correlation at early postoperative angiography. In addition, regression of the LITA graft stenosis accompanied the increase of the diameter of the LITA graft at follow-up angiography. These findings suggested that the increase of the LITA graft flow might result in regression of the LITA graft stenosis at the anastomotic site, and thus the LITA graft stenosis might be expandable. 
Thallium-201 stress scintigraphy was performed in 15 of 20 patients with $70 \%$ or greater diameter stenosis early after surgery, and only 2 patients showed reversible ischemia in the area of the coronary artery to which the LITA graft was anastomosed. Well-preserved native coronary artery flow might be a reason why ischemia was not detected through exercise. Furthermore, there is a possibility that patients could not get enough exercise because of their condition early after surgery. However, critical ischemic events did not occur at all early after surgery, despite leaving the LITA graft stenosis as it was. The increase in LITA graft flow during exercise might result in decrease of the LITA graft stenosis if the stenosis is expandable early after surgery.

\section{Intervention Therapy on the LITA Graft Stenosis at the Anastomotic Site}

Intervention therapy has been recently performed on the LITA graft stenosis at the anastomotic site early and late after surgery, and results of the intervention therapy on the LITA graft stenosis have been reported to be good. ${ }^{3,4}$ However, we should recognize the natural course of the LITA graft stenosis to pick up the patients who really need intervention therapy.

Our study demonstrated that LITA graft stenosis at the anastomotic site detected at early postoperative angiography might improve without intervention therapy. Critical ischemic events did not occur at all early after surgery, despite leaving the LITA graft stenosis as it was. No patients needed intervention therapy on the LITA graft late after surgery. Five patients with $70 \%$ or greater diameter stenosis early after surgery died during follow-up, but 4 of them showed regression of the LITA graft stenosis at follow-up angiography, which suggested that the deaths of the 4 patients were not related to LITA graft stenosis. One patient who did not show regression of the LITA graft stenosis at follow-up angiography resulted in sudden death 134 months after surgery. Therefore we conclude that LITA graft stenosis detected at early postoperative angiography might be observed without intervention therapy when the patients are asymptomatic or when ischemia is not detected through the exercise stress test. Intervention therapy might be considered when significant stenosis remains at follow-up angiography. Two patients with $90 \%$ or greater diameter stenosis early after surgery showed regression of the LITA graft stenosis at follow-up angiography and have been doing well without any cardiac events. Therefore it is difficult to identify patients who need intervention therapy on the LITA graft stenosis on the basis of the degree of stenosis at early postoperative angiography. In determining the indication of intervention therapy on the LITA graft, many factors must be considered, such as the degree of native coronary artery flow, the LITA graft diameter, myocardial viability, existence and extent of ischemic area, and so on. We think that it is important to recognize the natural course of the LITA graft stenosis as one of factors in considering intervention therapy.

Although routine postoperative angiography might not be performed today, recent reports ${ }^{19}$ have demonstrated that the LITA graft stenosis at the anastomotic site could be diagnosed by using transthoracic Doppler echocardiography. We think that it is important to know the natural course of the LITA graft stenosis in evaluating the LITA graft flow with transthoracic Doppler echocardiography.

\section{Study Limitations}

In our study early postoperative angiography was performed in 343 of 430 patients who underwent coronary bypass surgery by using the LITA graft, and follow-up angiography was performed in 100 of 343 patients who received postoperative angiography early after surgery. We retrospectively investigated early results of the LITA graft in the 343 patients and changes in the degree of the LITA graft stenosis in the 100 patients, and they underwent follow-up angiography for various reasons. Therefore our results might not represent the results of all patients who undergo coronary bypass surgery.

Quantitative angiographic analysis was applied to the anastomotic site. When the native coronary artery was patent, the results of quantitative angiography might not have been accurate.

\section{Conclusions}

In conclusion, LITA graft stenosis at the anastomotic site detected at early postoperative angiography might improve without intervention therapy. We should consider the natural course of the LITA graft stenosis in determining the indication of intervention therapy early after surgery.

\section{References}

1. Lytle BW, Loop FD, Cosgrove DM, Ratliff NB, Easley K, Taylor PC. Long-term (5 to 12 years) serial studies of internal mammary artery and saphenous vein coronary bypass grafts. J Thorac Cardiovasc Surg. 1985;89:248-58.

2. Loop FD, Lytle BW, Cosgrove DM, Stewart RW, Goormastic M, Williams GW, et al. Influence of the internal-mammary-artery graft on 10-year survival and other cardiac events. N Engl J Med. 1986;314:1-6.

3. Popma JJ, Cooke RH, Leon MB, Stark K, Satler LF, Kent KM, et al. Immediate procedural and long-term clinical results of internal mammary artery angioplasty. Am J Cardiol. 1992;69:1237-9.

4. Köckeritz U, Reynen K, Knaut M, Strasser RH. Results of angioplasty (with or without stent) at the site of a narrowed coronary anastomosis of the left internal mammary artery graft or via the internal mammary artery. Am J Cardiol. 2004;93:1531-3.

5. Sarabu MR, McClung JA, Fass A, Reed GE. Early postoperative spasm in left internal mammary artery bypass grafts. Ann Thorac Surg. 1987;44:199-200.

6. Izzat MB, West RR, Ragoonanan C, Angelini GD. Effect of systemic vasodilators on internal mammary artery flow. $J$ Thorac Cardiovasc Surg. 1994;108:82-5.

7. Sasson L, Cohen AJ, Hauptman E, Schachner A. Effect of topical vasodilators on internal mammary arteries. Ann Thorac Surg. 1995;59:494-6. 
8. Mills NL, Ochsner JL. Technique of internal mammary-to-coronary artery bypass. Ann Thorac Surg. 1974;17:237-46.

9. Jones EL, Lattouf OM, Weintraub WS. Catastrophic consequences of internal mammary artery hypoperfusion. J Thorac Cardiovasc Surg. 1989;98:902-7.

10. Segesser L, Simonet F, Meier B, Finci L, Faidutti B. Inadequate flow after internal mammary-coronary artery anastomoses. Thorac Cardiovasc Surg. 1987;35:352-4.

11. Seki T, Kitamura S, Kawachi K, Morita R, Kawata T, Mizuguchi K, et al. A quantitative study of postoperative luminal narrowing of the internal thoracic artery graft in coronary artery bypass surgery. $J$ Thorac Cardiovasc Surg. 1992;104:1532-8.

12. Geha AS, Baue AE. Early and late results of coronary revascularization with saphenous vein and internal mammary artery grafts. Am J Surg. 1979;137:456-63.

13. Ivert T, Huttunen K, Landou C, Björk VO. Angiographic studies of internal mammary artery grafts 11 years after coronary artery bypass grafting. J Thorac Cardiovasc Surg. 1988;96:1-12.

14. Dincer B, Barner HB. The "occluded" internal mammary artery graft:
Restoration of patency after apparent occlusion associated with progression of coronary disease. J Thorac Cardiovasc Surg. 1983;85:318-20.

15. Suma H. Internal thoracic artery and competitive flow. J Thorac Cardiovasc Surg. 1991;102:639-40.

16. Feld H, Navarro V, Kleeman H, Shani J. Early postoperative occlusion of a left internal mammary artery bypass graft with subsequent restoration of patency. Cathet Cardiovasc Diagn. 1992; 27:280-3.

17. Nakayama Y, Sakata R, Ura M. Growth potential of left internal thoracic artery grafts: analysis of angiographic findings. Ann Thorac Surg. 2001;71:142-7.

18. Berger PB, Alderman EL, Nadel A, Schaff HV. Frequency of early occlusion and stenosis in the left internal mammary artery to left anterior descending artery bypass graft after surgery through a median sternotomy on conventional bypass. Circulation. 1999;100: 2353-8.

19. Izumi C, Takahashi S, Kurozumi K, Hayashi H, Iga K, Miyake M, et al. Usefulness of high-frequency transthoracic Doppler echocardiography in noninvasive diagnosis of the left internal thoracic artery graft stenosis at the anastomotic site. Circ J. 2004;68:845-9. 